«Системні технологіï» 6 (125) 2019 «System technologies»

DOI 10.34185/1562-9945-6-125-2019-05

UDC 536.24

M.H. Berdnyk

\title{
MATHEMATIC SIMULATION OF GENERALIZED PROBLEM OF HEAT- EXCHANGE IN THE BODIES WITH HEMISPHERICAL SHAPE
}

Abstract. The article presents the first mathematical model for calculating temperature fields of hemispherical bodies, which approximately simulates operation of the diamond-drilling bit and takes into account angular velocity of drilling operations and finite velocity of heat conduction, and which was created as a physicomathematical boundary problem for hyperbolic equation of heat conduction with the Dirichlet boundary conditions. Besides, a new integral transformation was formulated for the two-dimensional finite space, with the help of which and with the help of finite element method and Galerkin method a temperature field was found in the form of convergence series.

Keywords: diamond-drilling bit, multiindex, integral transformation, finite element method.

Introduction. While estimating any territory by its potential hardmineral resources, the most critical problem is improvement of geological, technical and economic efficiency of drilling operations, which is impossible without up-to-date technical and technological support.

Well-drilling is the main method of mineral exploration, and diamond drilling with using of diamond-drilling bit is the most effective today and will be the most affordable in the future method for drilling wells in hard rock.

During the work, diamond-drilling bit is heavily heated due to the heat emission in the contact area and is cooled by a powerful stream of the drilling mud.

Taking account of the temperature effect creates a basis for predicting possible rate of the diamond-drilling mechanical velocity and choosing correct technological parameters and objectively controlling them during the drilling operations, and makes it possible to find proper ways for improving the diamond tool.

(C) Berdnyk M.H., 2019 
«Системні технологіï» 6 (125) 2019 «System technologies»

The issues of increasing of productivity and improving of quality and efficiency of drilling operations through the creation, implementation and improvement of diamond cutting tools are of continuing relevance.

Overview of the latest researches and publications. Analysis of papers [1-3] showed that most of the results are just partial, there is no common opinion about physics of drilling operations, and all of the known models do not allow predicting temperature of diamond-drilling bit heating during the operation with taking into account angular velocity of the drilling operations and ultimate velocity of the heat conduction.

Objective of the work. In order to simulate a temperature field, it is necessary to approximate shape of the bit to classical one and to accept some reasonable simplifications. In view of the small size of drilling diamonds in comparison with the size of the matrix, and their high thermal conductivity, let's assume that effect of diamond geometry is negligible.

Objective of this work was to construct a new mathematical model for calculating temperature fields of hemispherical body, which approximately simulates shape of the diamond bit, in the form of a boundary value problem of mathematical physics and to find solutions for this boundary value problem.

Presentation of the main research material. According to [4], contact temperature $t_{\kappa}$ on the surface of the bit can be represented as a linear dependence on the power $\mathrm{N}$ used for cutting:

$$
t_{k}=\left(k_{e}+\frac{1}{Q c_{p}}\right) k_{p} N+s \mathrm{H}+\mathrm{t}_{0}
$$

where $k_{e}$ is index of heat exchange intensity between diamond bit and washing fluid; $Q$ is mass consumption of washing fluid; $c_{p}$ is isobaric heat capacity of washing fluid; $k_{p}$ is coefficient of heat flow distribution between the rock and the bit; $H$ is current depth of the well; $s$ is thermal gradient coefficient; $t_{0}$ is temperature on the rock surface.

Let's consider calculation of temperature field of hemispherical body in cylindrical coordinate system $(r, \varphi, z)$ of radius $\mathrm{R}$ with generating line $r=\sqrt{R^{2}-(z-R)^{2}}$ and with center in the point $\left(0, \frac{\pi}{2}, R\right)$ (Fig.1). 


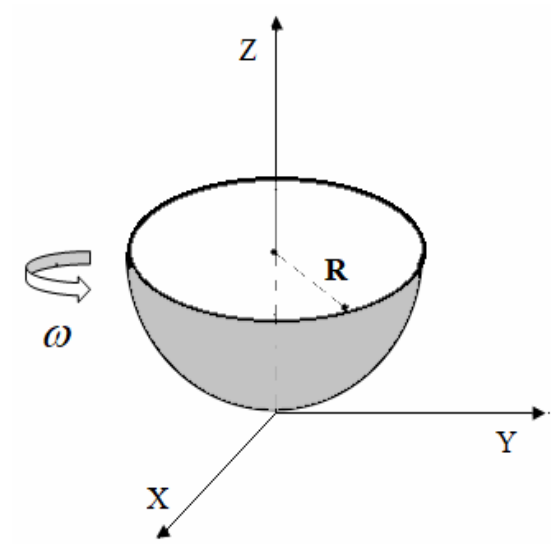

Figure 1- Hemispherical body with generating line $r=\sqrt{R^{2}-(z-R)^{2}}$

The body rotates around the $\mathrm{OZ}$ axis with constant angular velocity $\omega$, and rate of heat conduction is known. Heat-transfer properties of the body do not depend on temperature, and internal sources of heat are not available. At the initial time, the body temperature $G_{0}$ is constant, and, according to (1), temperature $V(\varphi, z)$ on the lateral surface is known and does not depend on time. At $\mathrm{z}=\mathrm{R}$, value of temperature $G_{1}(r, \varphi)$ is known.

In [5], a generalized energy transfer equation was obtained for a moving element of continuous medium with taking into account finiteness of heat conduction velocity. According to [5], generalized equation of energy balance in the solid, which rotates around the $\mathrm{OZ}$ axis with constant angular velocity $\omega$, and whose thermophysical properties do not depend on temperature, and no internal heat sources are available, is expressed in cylindrical coordinate system as:

$$
\gamma \mathrm{c}\left\{\frac{\partial \mathrm{T}}{\partial \mathrm{t}}+\omega \frac{\partial T}{\partial \varphi}+\tau_{r}\left[\frac{\partial^{2} T}{\partial t^{2}}+\omega \frac{\partial^{2} T}{\partial \varphi \partial t}\right]\right\}=\lambda\left[\frac{\partial^{2} T}{\partial r^{2}}+\frac{1}{r} \frac{\partial T}{\partial r}+\frac{1}{r^{2}} \frac{\partial^{2} T}{\partial \varphi^{2}}+\frac{\partial^{2} T}{\partial z^{2}}\right]
$$

where $\gamma$-is density of the medium; $c$ is specific heat capacity; $\tau_{r}$ is relaxation time; $T(\rho, \varphi, z, t)$ is temperature of the medium; $\lambda$ - is heat conductivity coefficient; $t$ is time.

In terms of mathematics, problem of determining temperature field of the body consists in integrating differential equation of heat conductivity (2) into the domain $D=\left\{(\rho, \varphi, z, t) \mid \rho \in\left(0, \sqrt{1-(z-1)^{2}}\right), \varphi \in(0,2 \pi), \mathrm{z} \in(0,1), \mathrm{t} \in(0, \infty)\right\}$, which, with taking into account the accepted assumptions, will be written as: 
«Системні технологіï» 6 (125) 2019 «System technologies»

$$
\frac{\partial \theta}{\partial t}+\omega \frac{\partial \theta}{\partial \varphi}+\tau_{r} \frac{\partial^{2} \theta}{\partial t^{2}}+\tau_{r} \omega \frac{\partial^{2} \theta}{\partial \varphi \partial t}=\frac{a}{R^{2}}\left[\frac{\partial^{2} \theta}{\partial \rho^{2}}+\frac{1}{\rho} \frac{\partial \theta}{\partial \rho}+\frac{1}{\rho^{2}} \frac{\partial^{2} \theta}{\partial \varphi^{2}}+\frac{\partial^{2} \theta}{\partial z^{2}}\right]
$$

with initial conditions

$$
\theta(\rho, \varphi, z, 0)=0, \quad \frac{\partial \theta(\rho, \varphi, z, 0)}{\partial t}=0
$$

and with boundary conditions

$$
\begin{gathered}
\theta\left(\sqrt{1-(z-1)^{2}}, \varphi, z, t\right)=G(\varphi, z), \\
\theta(\rho, \varphi, 1, t)=\Lambda(\rho, \varphi),
\end{gathered}
$$

where $\theta=\frac{T(\rho, \varphi, z, t)-G_{0}}{T_{\max }-G_{0}}$ is relative temperature of the body; $a=\frac{\lambda}{c \gamma}$ is heat conductivity coefficient; $\quad T_{\max }=\max \left\{V(\varphi, z), G_{1}(r, \varphi)\right\} ; \quad \rho=\frac{r}{R} ; \quad z=\frac{z}{R}$; $G(\varphi, z), \Lambda(\rho, \varphi) \in \mathrm{C}(0,2 \pi)$.

In this case, solution of the boundary problem (3)-(6) $\theta(\rho, \varphi, z, t)$ is twice continuously differentiated by $\rho$ and $\varphi$, z, is once differentiated by $t$ in the domain $\mathrm{D}$ and is continuous on the $\bar{D}[6]$, i.e. $\theta(\rho, \varphi, z, t) \in C^{2,1}(D) \cap C(\bar{D})$, and functions $G(\varphi, z), \Lambda(\rho, \varphi), \theta(\rho, \varphi, z, t)$ can be expressed by the Fourier complex series [6]

$$
\left\{\begin{array}{c}
\theta(\rho, \varphi, z, t) \\
G(\varphi, z) \\
\Lambda(\rho, \varphi)
\end{array}\right\}=\sum_{n=-\infty}^{+\infty}\left\{\begin{array}{c}
\theta_{n}(\rho, z, t) \\
G_{n}(z) \\
\Lambda_{n}(\rho)
\end{array}\right\} \cdot \exp (\operatorname{in} \varphi)
$$

where

$$
\left\{\begin{array}{c}
\theta_{n}(\rho, z, t) \\
G_{n}(z) \\
\Lambda_{n}(\rho,)
\end{array}\right\}=\frac{1}{2 \pi} \int_{0}^{2 \pi}\left\{\begin{array}{c}
\theta(\rho, \varphi, z, t) \\
G(\varphi, z) \\
\Lambda(\rho, \varphi)
\end{array}\right\} \cdot \exp (-i n \varphi) d \varphi,
$$

$\theta_{n}(\rho, z, t)=\theta_{n}^{(1)}(\rho, z, t)+i \theta_{n}^{(2)}(\rho, z, t), \mathrm{G}_{n}(z)=G_{n}^{(1)}(z)+i G_{n}^{(2)}(z), \Lambda_{n}(\rho)=\Lambda_{n}^{(1)}(\rho)+i \Lambda_{n}^{(2)}(\rho)$.

In view of the fact that $\theta(\rho, \varphi, z, t)$ is a real-valued function, let's confine ourselves by considering only $\theta_{n}(\rho, z, t)$ for $\mathrm{n}=0,1,2, \ldots$, because $\theta_{n}(\rho, z, t)$ and $\theta_{-n}(\rho, z, t)$ are complexly conjugate [6]. By putting values of functions from (7) into (3)-(6) we receive the following system of differential equations: 
«Системні технологіï» 6 (125) 2019 «System technologies»

$$
\frac{\partial \theta_{n}^{(i)}}{\partial t}+\vartheta_{n}^{(i)} \theta_{n}^{\left(m_{i}\right)}+\tau_{r} \frac{\partial^{2} \theta_{n}^{(i)}}{\partial t^{2}}+\tau_{r} \vartheta_{n}^{(i)} \frac{\partial \theta_{n}^{\left(m_{i}\right)}}{\partial t}=\frac{a}{R^{2}}\left[\frac{\partial^{2} \theta_{n}^{(i)}}{\partial \rho^{2}}+\frac{1}{\rho} \frac{\partial \theta_{n}^{(i)}}{\partial \rho}-\frac{n^{2}}{\rho^{2}} \theta_{n}^{(i)}+\frac{\partial^{2} \theta_{n}^{(i)}}{\partial z^{2}}\right]
$$

with initial conditions

$$
\theta_{n}^{(i)}(\rho, z, 0)=0, \quad \frac{\partial \theta_{n}^{(i)}(\rho, z, 0)}{\partial t}=0
$$

and with boundary conditions

$$
\begin{gathered}
\theta_{n}^{(i)}\left(\sqrt{1-(z-1)^{2}}, z, t\right)=G_{n}^{(i)}(z) \\
\theta_{n}^{(i)}(\rho, 1, t)=\Lambda_{n}^{(i)}(\rho),
\end{gathered}
$$

where $\vartheta_{n}^{(1)}=-\omega n ; \quad \vartheta_{n}^{2}=\omega n ; \mathrm{m}_{1}=2, \mathrm{~m}_{2}=1 ; \quad \mathrm{i}=1,2$.

In order to solve the boundary problem (8)-(11), let's employ the integral transformation:

$$
\bar{f}\left(\mu_{n, k}\right)=\iint_{\Xi} \phi\left(\rho, z, \mu_{n, k}\right) \cdot \rho \cdot \mathrm{f}(\rho, z) d \sigma
$$

where $\phi\left(x, y, \mu_{n, k}\right), \mu_{n, k}$ are intrinsic functions and intrinsic values.

Classical problem of intrinsic values and intrinsic functions is formulated as a problem of defining values for numerical parameters (intrinsic values) $\mu_{n, k}$ and functions (intrinsic functions) $\phi\left(x, y, \mu_{n, k}\right)$, which are identically not equal to zero in the domain $\Xi=\left\{(x, y) \mid \mathrm{y} \in(0,1), x \in\left(0, \sqrt{1-(z-1)^{2}}\right)\right\}$, and satisfy the equation:

$$
\frac{\partial^{2} \phi}{\partial x^{2}}+\frac{1}{x} \frac{\partial \phi}{\partial x}-\frac{n^{2}}{x^{2}} \phi+\mu_{n, k} \cdot \phi+\frac{\partial^{2} \phi}{\partial y^{2}}=0
$$

and subsidiary conditions

$$
\begin{gathered}
\phi\left(\sqrt{1-(z-1)^{2}}, y, \mu_{n, k}\right)=0, \\
\phi\left(x, 1, \mu_{n, k}\right)=0
\end{gathered}
$$

where $\quad \phi\left(x, y, \mu_{n, k}\right) \subset C^{2}(\Xi)=\left\{u(x, y) \in C(\Xi): \partial_{\alpha} u(x, y) \in C(\Xi), \forall \alpha,|\alpha| \leq 2\right\}$; $\partial_{\alpha} u(x, y)=\frac{\partial^{|\alpha|} u(x, y)}{\partial x^{\alpha_{1}} \partial x_{2}^{\alpha_{2}}} ;|\alpha|=\alpha_{1}+\alpha_{2}-$ is multiindex, components of which are whole integral numbers.

Let's find intrinsic values $\mu_{n, k}$ and intrinsic functions $\phi\left(x, y, \mu_{n, k}\right)$ by solving problems (13) - (15) with the help of finite element methods and 
Galerkin method. To this end, let's divide the domain into simplex elements (Fig. 2):

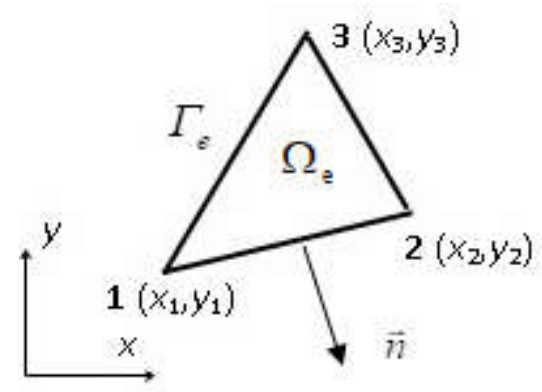

Figure 2 - Triangular element of the first order

Then, function $\phi(x, y)$ inside the simplex element is expressed through the shape functions, $N_{1}, N_{2}$ and $N_{3}$ with the known values of $\phi_{1}, \phi_{2}, \phi_{3}$ in the vertices of the triangle:

$$
\phi_{e}(x, y)=N_{1} \phi_{1}+N_{2} \phi_{2}+N_{3} \phi_{3}=\left[N_{e}\right]^{T}\left\{\phi_{e}\right\}
$$

where $\left[N_{e}\right]=\left[N_{1}, N_{2}, N_{3}\right]^{T} ;\left\{\phi_{e}\right\}=\left\{\phi_{1}, \phi_{2}, \phi_{3}\right\}^{T}$; inferior index (e) means free simplex element.

For the $i$-th node $(i=1,2,3)$, the shape functions are expressed as:

$$
N_{i}(x, y)=\frac{1}{d}\left(a_{i}+b_{i} x+c_{i} y\right)
$$

where $a_{i}=x_{j} y_{k}-x_{k} y_{j} ; b_{i}=y_{j}-y_{k} ; ; c_{i}=x_{k}-x_{j}$; $d=x_{2} y_{3}-x_{3} y_{2}+x_{3} y_{1}-x_{1} y_{3}+x_{1} y_{2}-x_{2} y_{1} ; i, j, k$ is sequent numbering of nodes in the simplex element while bypassing them counterclockwise.

In order to determine shape functions $N_{1}, N_{2}, N_{3}$, it is convenient to use coordinate $L_{i}$ inside the simplex element, which is determined by relation of area of the triangle created by the point and side of the opposite vertex $i$, to general area of the triangle:

$$
\begin{aligned}
& L_{1}=\frac{1}{d} \cdot\left[\left(y_{2}-y_{3}\right)\left(x-x_{2}\right)+\left(x_{3}-x_{2}\right)\left(y-y_{2}\right)\right] ; \\
& L_{2}=\frac{1}{d} \cdot\left[\left(y_{3}-y_{1}\right)\left(x-x_{3}\right)+\left(x_{1}-x_{3}\right)\left(y-y_{3}\right)\right] ; \\
& L_{3}=\frac{1}{d} \cdot\left[\left(y_{1}-y_{2}\right)\left(x-x_{1}\right)+\left(x_{2}-x_{1}\right)\left(y-y_{1}\right)\right] .
\end{aligned}
$$


«Системні технологіï» 6 (125) 2019 «System technologies»

In case of linear simplex element, which includes three vertexes, shape functions are in harmony with corresponding coordinate $L_{i}$ :

$$
N_{1}=L_{1}, \quad N_{2}=L_{2}, \quad N_{3}=L_{3} .
$$

Let's put the approximate solution (16) into equation (13) and, as a result, we obtain the following equation:

$$
\left(\frac{\partial^{2}}{\partial x^{2}}+\frac{1}{x} \frac{\partial}{\partial x}+\frac{\partial^{2}}{\partial y^{2}}\right)\left[N_{e}\right]^{T}\left\{\phi_{e}\right\}+\left(\mu_{n, k}-\frac{n^{2}}{x^{2}}\right)\left[N_{e}\right]^{T}\left\{\phi_{e}\right\}=0
$$

Multiplying of the left side of the equation (17) by shape function $\left[N_{e}\right]$ and integrating by element $e$ will give:

$$
I_{1}+I_{2}=\{0\}
$$

where

$$
I_{1}=\iint_{\Omega_{\mathrm{e}}}\left[N_{e}\right]\left(\frac{\partial^{2}}{\partial x^{2}}+\frac{1}{x} \frac{\partial}{\partial x}+\frac{\partial^{2}}{\partial y^{2}}\right)\left[N_{e}\right]^{T} d x d y\left\{\phi_{e}\right\} ; I_{2}=\iint_{\Omega_{\mathrm{e}}}\left(\mu_{n, k}-\frac{n^{2}}{x^{2}}\right)\left[N_{e}\right]\left[N_{e}\right]^{T} d x d y\left\{\phi_{e}\right\} \text {. }
$$

By integrating $\mathrm{I}_{1}$ along the $x$ and $y$, we receive:

$$
\begin{gathered}
I_{1}=I_{3}-I_{4}, \\
I_{3}=\left[\int_{\Gamma_{e}}\left[N_{e}\right] \frac{\partial\left[N_{e}\right]^{T}}{\partial x} d y+\int_{\Gamma_{e}}\left[N_{e}\right] \frac{\partial\left[N_{e}\right]^{T}}{\partial y} d x\right]\left\{\phi_{e}\right\}, \\
I_{4}=\iint_{\Omega_{\mathrm{e}}}\left\{\frac{\partial\left[N_{e}\right]}{\partial x} \frac{\partial\left[N_{e}\right]^{T}}{\partial x}+\frac{\partial\left[N_{e}\right]}{\partial y} \frac{\partial\left[N_{e}\right]^{T}}{\partial y}-\frac{\left[N_{e}\right]}{x} \frac{\partial\left[N_{e}\right]^{T}}{\partial x}\right\} d x d y\left\{\phi_{e}\right\} .
\end{gathered}
$$

By taking into account identical relation

$$
\int_{\Gamma} \psi \frac{\partial \varphi}{\partial x} d y+\int_{\Gamma} \psi \frac{\partial \varphi}{\partial y} d x=\int_{\Gamma} \psi\left(\frac{\partial \varphi}{\partial x} d y+\frac{\partial \varphi}{\partial y} d x\right)=\int_{\Gamma} \psi \frac{\partial \varphi}{\partial n} d \Gamma
$$

we receive

$$
I_{3}=\left[\int_{\Gamma_{e}}\left[N_{e}\right] \frac{\partial\left[N_{e}\right]^{T}}{\partial n} d \Gamma\right]\left\{\phi_{e}\right\},
$$

where $\partial / \partial n$ is outer normal derivative; $\int_{\Gamma} d \Gamma$ is boundary curvilinear integral. Summing of all elements will give us:

$$
\begin{aligned}
& \sum_{e}\left[\int_{\Gamma_{e}}\left[N_{e}\right] \frac{\partial\left[N_{e}\right]^{T}}{\partial n} d \Gamma\right]\left\{\phi_{e}\right\}+\sum_{e} \iint_{\Omega_{e}}\left(\mu_{n}-\frac{n^{2}}{x^{2}}\right)\left[N_{e}\right]\left[N_{e}\right]^{T} d x d y\left\{\phi_{e}\right\}- \\
& -\sum_{e} \iint_{\Omega_{e}}\left\{\frac{\partial\left[N_{e}\right]}{\partial x} \frac{\partial\left[N_{e}\right]^{T}}{\partial x}+\frac{\partial\left[N_{e}\right]}{\partial y} \frac{\partial\left[N_{e}\right]^{T}}{\partial y}-\frac{\left[N_{e}\right]}{x} \frac{\partial\left[N_{e}\right]^{T}}{\partial x}\right\} d x d y\left\{\phi_{e}\right\}=\{0\}
\end{aligned}
$$


«Системні технології» 6 (125) 2019 «System technologies»

By multiplying the augend (18) by the expression $\left\{\phi_{e}\right\}\left\{\phi_{e}\right\}^{T}$ we receive:

$$
\begin{aligned}
& \left.I_{3}=\sum_{e} \phi_{e}\right\}^{T}\left[\int_{\Gamma_{e}}\left[N_{e}\right] \frac{\partial\left[N_{e}\right]^{T}}{\partial n} d \Gamma\right]\left\{\phi_{e}\right\}=\sum_{e}\left[\int_{\Gamma_{e}}\left(\left\{\phi_{e}\right\}^{T}\left[N_{e}\right]\right) \cdot \frac{\partial\left(\left[N_{e}\right]^{T}\left\{\phi_{e}\right\}\right)}{\partial n} d \Gamma\right]= \\
& \sum_{e}\left[\int_{\Gamma_{e}} \phi_{e} \frac{\partial \phi_{e}}{\partial n} d \Gamma\right] .
\end{aligned}
$$

In this case, the boundary curvilinear integrals $\Gamma_{\mathrm{e}}$ of elements inside the domain $\Xi$ is reduced because, while calculating the curvilinear integrals, directions of bypassing the boundary are opposite for each pair of the neighbor elements (Fig. 3).

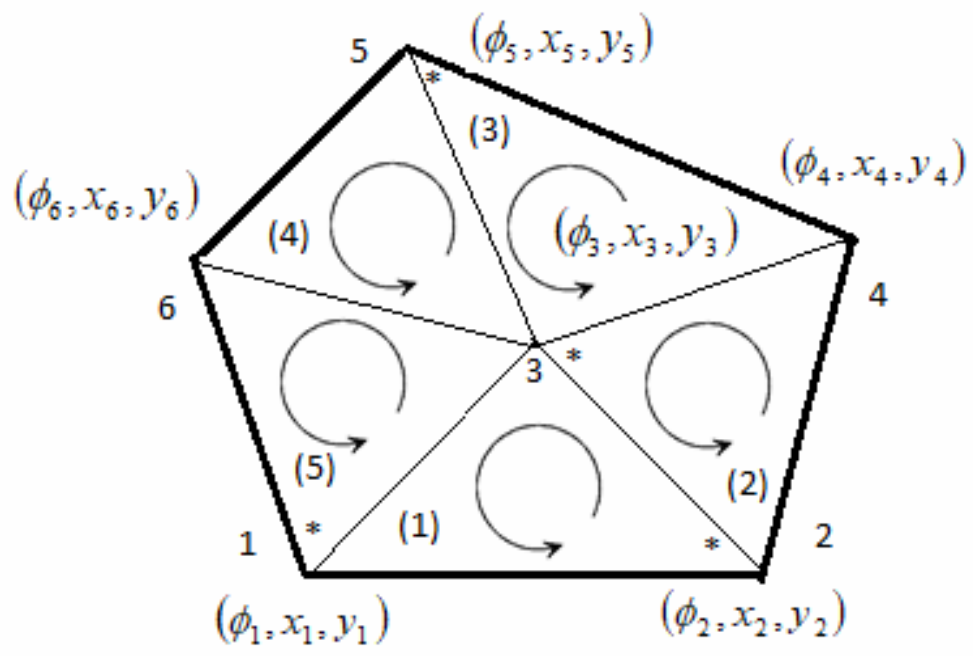

Figure 3 - Directions of bypassing the boundary inside the domain $\Xi$

As a result, only the curvilinear integral remains on the boundary $\Gamma$ of the entire domain $\Xi$ Although such an assumption is one of the limitations for the finite element method, it is a relatively good approximation. Then, taking into account the Dirichlet boundary condition, one can ignore the first term in (18).

In this case, (18) takes the following form:

$$
[K]+\mu_{n, k} \cdot[M]=\{0\}
$$

where

$[K]=\sum_{e}\left\{-\iint_{e}\left(\frac{\partial\left[N_{e}\right]}{\partial x} \frac{\partial\left[N_{e}\right]^{T}}{\partial x}+\frac{\partial\left[N_{e}\right]}{\partial y} \frac{\partial\left[N_{e}\right]^{T}}{\partial y}-\frac{\left[N_{e}\right]}{x} \frac{\partial\left[N_{e}\right]^{T}}{\partial x}\right) d x d y-\frac{n^{2}}{x^{2}} \iint_{e}\left[N_{e}\right]\left[N_{e}\right]^{T} d x d y\right\} \phi_{e}$ 
«Системні технологіï» 6 (125) 2019 «System technologies»

$$
[M]=\sum_{e} \iint_{e}\left[N_{e}\right]\left[N_{e}\right]^{T} d x d y \cdot\left\{\phi_{e}\right\} .
$$

Therefore, intrinsic functions $\phi\left(x, y, \mu_{n, k}\right)$ and intrinsic values $\mu_{n, k}$ can be found from (19), and formula of inverse transformation takes the following form:

$$
\mathrm{f}(\rho, z)=\sum_{j=1}^{\infty} \frac{\phi\left(\rho, z, \mu_{n, k}\right)}{\left\|\phi\left(\rho, z, \mu_{n, k}\right)\right\|^{2}} \bar{f}\left(\mu_{n, k}\right) .
$$

Let's employ the integral transformation (12) for the system of differential equations (8), and, as a result, we receive the following system of ordinary differential equations:

$$
\frac{d \bar{\theta}_{n}^{(i)}}{d t}+\vartheta_{n}^{(i)}\left[\bar{\theta}_{n}^{\left(m_{i}\right)}+\tau_{r} \frac{d \bar{\theta}_{n}^{\left(m_{i}\right)}}{d t}\right]+\tau_{r} \frac{d^{2} \bar{\theta}_{n}^{(i)}}{d t^{2}}=\frac{a}{R^{2}}\left(\Omega_{\mathrm{n}, \mathrm{k}}^{(\mathrm{i})}-\mu_{n, k} \bar{\theta}_{n}^{(i)}\right)
$$

with initial conditions

$$
\bar{\theta}_{n}^{(i)}\left(\mu_{n, k}, t\right)=0, \quad \frac{\partial \bar{\theta}_{n}^{(i)}\left(\mu_{n, k}, t\right)}{\partial t}=0,
$$

where

$$
\Omega_{\mathrm{n}, \mathrm{k}}^{(\mathrm{i})}=-\int_{0}^{1} \sqrt{1-(z-1)^{2}} \cdot \frac{\partial \phi\left(\sqrt{1-(z-1)^{2}}, z, \mu_{n, k}\right)}{\partial \rho} G_{n}^{(i)}(z) d z-\oint_{L} \rho \theta_{n}^{(i)} \frac{\partial \phi\left(\rho, z, \mu_{n, k}\right)}{\partial z} d \rho .
$$

The curvilinear integral is calculated by the closed positively-oriented contour (Fig. 4):

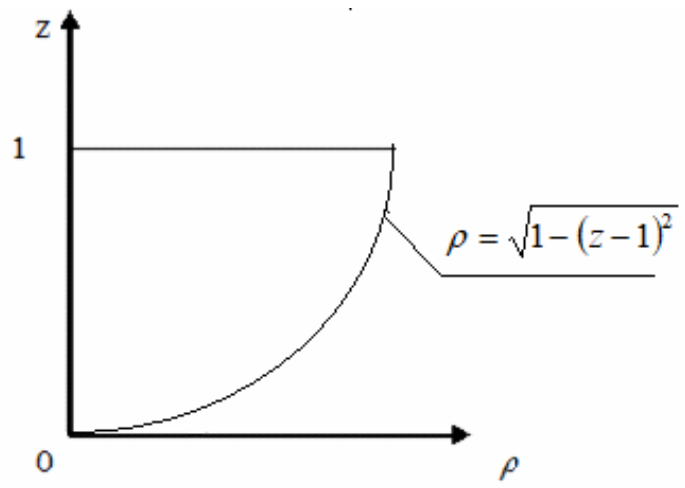

Figure 4 - Closed contour with generating line: $\rho=\sqrt{1-(z-1)^{2}}$

Let's apply the Laplace integral transformation [7] with initial conditions of (22) to the system of integral equations (21):

$$
\widetilde{f}(s)=\int_{0}^{\infty} f(\tau) e^{-s \tau} \mathrm{d} \tau
$$


«Системні технології» 6 (125) 2019 «System technologies»

As a result we receive a system of algebraic equations relatively to the $\widetilde{\bar{\theta}}_{n}^{(i)}$

$$
s \widetilde{\bar{\theta}}_{n}^{(i)}+\vartheta_{n}^{(i)}\left(\widetilde{\bar{\theta}}_{n}^{\left(m_{i}\right)}+\tau_{r} s \widetilde{\bar{\theta}}_{n}^{\left(m_{i}\right)}\right)+\tau_{r} s^{2} \widetilde{\bar{\theta}}_{n}^{(i)}=q_{n, k}\left(\frac{\widetilde{\Omega}_{\mathrm{n}, \mathrm{k}}^{(\mathrm{i})}}{\mu_{n, k}}-\widetilde{\bar{\theta}}_{n}^{(i)}\right)
$$

where $\mathrm{i}=1,2 ; q_{n, k}=\frac{\mathrm{a}}{\mathrm{R}^{2}} \mu_{n, k}$.

Having solved the system of equations (23) we receive:

$$
\widetilde{\bar{\theta}}_{n}^{(i)}=\alpha_{n, \mathrm{k}} \frac{\widetilde{\Omega}_{\mathrm{n}, \mathrm{k}}^{(\mathrm{i})}\left(\tau_{r} s^{2}+s+\mathrm{q}_{\mathrm{n}, \mathrm{k}}\right)+(-1)^{i+1} \omega n \widetilde{\Omega}_{\mathrm{n}, \mathrm{k}}^{\left(m_{i}\right)}\left(1+s \tau_{r}\right)}{\left(\tau_{r} s^{2}+s+\mathrm{q}_{\mathrm{n}, \mathrm{k}}\right)^{2}+\omega^{2} n^{2}\left(1+s \tau_{r}\right)^{2}},
$$

where $\alpha_{n, k}=\frac{\mathrm{a}}{\mathrm{R}^{2}} ;(i=1,2$.

By applying the Laplace formula of inverse transformation for the expression of function (24), we receive the following original functions:

$$
\begin{aligned}
& \bar{\theta}_{\mathrm{n}}^{(1)}\left(\mu_{\mathrm{n}, \mathrm{k}}, \mathrm{t}\right)=\sum_{\mathrm{j}=1}^{2} \zeta_{\mathrm{n}, \mathrm{k}}\left(\mathrm{s}_{\mathrm{j}}\right)\left\{\tilde{\Omega}_{\mathrm{n}, \mathrm{k}}^{(1)}\left(\mathrm{s}_{\mathrm{j}}\right) \cdot\left[\left(2 \tau_{\mathrm{r}} \mathrm{s}_{\mathrm{j}}+1\right)+\tau_{\mathrm{r}} \omega \mathrm{ni}\right]+\tilde{\Omega}_{\mathrm{n}, \mathrm{k}}^{(2)}\left(\mathrm{s}_{\mathrm{j}}\right) \cdot\left[\tau_{\mathrm{r}} \omega \mathrm{n}-\left(2 \tau_{\mathrm{r}} \mathrm{s}_{\mathrm{j}}+1\right) \mathrm{i}\right]\right\} \cdot \\
& \left(\mathrm{e}^{\mathrm{s}_{\mathrm{j}} \mathrm{t}}-1\right)+\sum_{\mathrm{j}=3}^{4} \zeta_{\mathrm{n}, \mathrm{k}}\left(\mathrm{s}_{\mathrm{j}}\right) \cdot\left\{\tilde{\Omega}_{\mathrm{n}, \mathrm{k}}^{(1)}\left(\mathrm{s}_{\mathrm{j}}\right) \cdot\left[\left(2 \tau_{\mathrm{r}} \mathrm{s}_{\mathrm{j}}+1\right)-\tau_{\mathrm{r}} \omega \mathrm{ni}\right]+\tilde{\Omega}_{\mathrm{n}, \mathrm{k}}^{(2)}\left(\mathrm{s}_{\mathrm{j}}\right) \cdot\left[\tau_{\mathrm{r}} \omega \mathrm{n}+\left(2 \tau_{\mathrm{r}} \mathrm{s}_{\mathrm{j}}+1\right) \mathrm{i}\right]\right\}, \\
& \cdot\left(\mathrm{e}^{\mathrm{s}_{\mathrm{j}} \mathrm{t}}-1\right),
\end{aligned}
$$

$\bar{\theta}_{\mathrm{n}}^{(2)}\left(\mu_{\mathrm{n}, \mathrm{k}}, \mathrm{t}\right)=\sum_{\mathrm{j}=1}^{2} \zeta_{\mathrm{n}, \mathrm{k}}\left(\mathrm{s}_{\mathrm{j}}\right) \cdot\left\{\tilde{\Omega}_{\mathrm{n}, \mathrm{k}}^{(2)}\left(\mathrm{s}_{\mathrm{j}}\right) \cdot\left[\left(2 \tau_{\mathrm{r}} \mathrm{s}_{\mathrm{j}}+1\right)+\tau_{\mathrm{r}} \omega \mathrm{ni}\right]-\tilde{\Omega}_{\mathrm{n}, \mathrm{k}}^{(1)}\left(\mathrm{s}_{\mathrm{j}}\right) \cdot\left[\tau_{\mathrm{r}} \omega \mathrm{n}-\left(2 \tau_{\mathrm{r}} \mathrm{s}_{\mathrm{j}}+1\right) \mathrm{i}\right]\right\} \cdot$

$\left(\mathrm{e}^{\mathrm{s}_{\mathrm{j}} \mathrm{t}}-1\right)+\sum_{\mathrm{j}=3}^{4} \zeta_{\mathrm{n}, \mathrm{k}}\left(\mathrm{s}_{\mathrm{j}}\right) \cdot\left\{\tilde{\Omega}_{\mathrm{n}, \mathrm{k}}^{(2)}\left(\mathrm{s}_{\mathrm{j}}\right) \cdot\left[\left(2 \tau_{\mathrm{r}} \mathrm{s}_{\mathrm{j}}+1\right)-\tau_{\mathrm{r}} \omega \mathrm{ni}\right]-\tilde{\Omega}_{\mathrm{n}, \mathrm{k}}^{(1)}\left(\mathrm{s}_{\mathrm{j}}\right) \cdot\left[\tau_{\mathrm{r}} \omega \mathrm{n}+\left(2 \tau_{\mathrm{r}} \mathrm{s}_{\mathrm{j}}+1\right) \mathrm{i}\right]\right\}$ $\left(\mathrm{e}^{\mathrm{s}_{\mathrm{j}}^{\mathrm{t}}}-1\right)$

where $\zeta_{n, k}\left(s_{j}\right)=\frac{0.5 s_{j}^{-1} \alpha_{n, k}}{\left(2 \tau_{r} s_{j}+1\right)^{2}+\left(\tau_{r} \omega n\right)^{2}}$, and values of $s_{j}$ for $\mathrm{j}=1,2,3,4$ are determined by the formulas 
«Системні технологіï» 6 (125) 2019 «System technologies»

$s_{1,2}=\frac{\left(\tau_{r} \omega n i-1\right) \pm \sqrt{\left(1+\tau_{r} \omega n i\right)^{2}-4 \tau_{r} q_{n, k}}}{2 \tau_{r}}, s_{, 3,4}=\frac{\left(\tau_{r} \omega n i+1\right) \pm \sqrt{\left(1-\tau_{r} \omega n i\right)^{2}-4 \tau_{r} q_{n, k}}}{2 \tau_{r}}$.

By this way, and by taking into account formulas of inverse transformation (7) and (20), we receive a temperature field of hemispherical body, which rotates around the $\mathrm{OZ}$ axis with constant angular velocity $\omega$, with taking into account finite velocity of the heat conductivity:

$$
\theta(\rho, \varphi, z, t)=\sum_{n=-\infty}^{+\infty}\left\{\sum_{\mathrm{k}=1}^{\infty}\left[\bar{\theta}_{n}^{(1)}\left(\mu_{n, k}, t\right)+i \cdot \bar{\theta}_{n}^{(2)}\left(\mu_{n, k}, t\right)\right] \frac{\phi\left(\rho, z, \mu_{n, k}\right)}{\left\|\phi\left(\rho, z, \mu_{n, k}\right)\right\|^{2}}\right\} \cdot \exp (\operatorname{in} \varphi),
$$

where values for $\bar{\theta}_{n}^{(1)}\left(\mu_{n, k}, t\right), \bar{\theta}_{n}^{(2)}\left(\mu_{n, k}, t\right)$ are determined by formulas (25),(26).

Conclusions. It is the first mathematical model developed for calculating temperature fields of hemispherical body, which approximately simulates operation of the diamond-drilling bit and takes into account angular velocity of drilling operations and finite velocity of heat conduction, and which was created as a physicomathematical boundary problem for hyperbolic equation of heat conduction with the Dirichlet boundary conditions.

Besides, a new integral transformation was created for the twodimensional finite space, with the help of which and with the help of finite element method and Galerkin method a temperature field was found in the form of convergence series.

The obtained solution can be used for predicting possible rate of the diamond-drilling mechanical velocity, choosing correct technological parameters and for objectively controlling them during the drilling operations; besides, it makes possible to find proper ways for improving the diamond-bit operation.

\section{ЛИТЕРАТУРА / ЛІТЕРАТУРА}

1. Александров В. А. Температурное поле и износ неоднородного алмазного круга при конвективном теплообмене / В.А.Александров, А.Н. Жуковский, В. А. Мечник // Трение и износ. - 1994. -Т. 15, - № 1. - С. 27- 35. 2. Бондаренко Н.А. Температурное состояние алмазных буровых долот/ Н.А.Бондаренко, А.Н. Жуковский, В.А. Мечник // Доповіді НАН України. - 2006. - № 10. - С. 95 - 102. 
«Системні технологіï» 6 (125) 2019 «System technologies»

3. Кожевников А.А. Математическая модель температурного режима алмазной коронки при бурении скважины / А.А. Кожевников, А.Ю. Дреус, И.И. Мартыненко, Ю.А. Бакаржиев // Матеріали міжнар. конф. “Форум гірників-2005”.- Дніпропетровськ: НДУ, 2005. - Т. 2. - С. 250-257.

4. Горшков Л.К. Температурные режимы алмазного бурения/Л.К. Горшков, В.Г. Гореликов. - М.: Недра, 1992. - 212 с.

5. Бердник М.Г. Математичне моделювання температурного поля в циліндрі, який обертається, 3 урахуванням кінцевої швидкості поширення тепла / М.Г. Бердник // Питання прикладної математики і математичного моделювання. - Дніпропетровськ: ДНУ, 2005. - С. 37-44.

6. Маркович Б. М. Рівняння математичної фізики/Б.М. Маркович. - Львів: Львівська політехніка, 2010. - 384 с.

7. Лопушанська Г.П. Перетворення Фур’є, Лапласа: узагальнення та застосування/ Г.П. Лопушанська, А.О. Лопушанський, О.М. М’яус. - Львів: ЛНУ ім. Івана Франка, 2014. -152 с.

\section{REFERENCES}

1. Aleksandrov V. A. Temperaturnoe pole i iznos neodnorodnogo almaznogo kruga pri konvektivnom teploobmene / V.A.Aleksandrov, A.N. Zhukovskij, V. A. Mechnik // Trenie i iznos. - 1994. - T. 15, - № 1. - S. 27 - 35.

2. Bondarenko N.A. Temperaturnoe sostojanie almaznyh burovyh dolot/ N.A.Bondarenko, A.N. Zhukovskij, V.A.Mechnik, // Dopovidi NAN Ukraïni. 2006. - № 10. - S. 95 - 102.

3. Kozhevnikov A.A. Matematicheskaja model' temperaturnogo rezhima almaznoj koronki pri burenii skvazhiny / A.A. Kozhevnikov, A.Ju. Dreus, I.I. Martynenko, Ju.A. Bakarzhiev // Materiali mizhnar. konf. “Forum girnikiv2005”.-Dnipropetrovs'k: NDU, 2005. - T. 2. -S. 250-257.

4. Gorshkov L.K. Temperaturnye rezhimy almaznogo burenija/L.K. Gorshkov, V.G. Gorelikov. - M.: Nedra, 1992. - 212 s.

5. Berdnik M.G. Matematichne modeljuvannja temperaturnogo polja V cilindri, jakij obertaєt'sja, z urahuvannjam kincevoï shvidkosti poshirennja tepla / M.G. Berdnik // Pitannja prikladnoï matematiki i matematichnogo modeljuvannja. - Dnipropetrovs'k: DNU, 2005. - S. 37-44. 
6. Markovich B. M. Rivnjannja matematichnoï fiziki/B. M. Markovich. - L'viv: L'vivs'ka politehnika, 2010. - 384 c.

7. Lopushanska, G.P. Peretvorennja Furje, Laplasa: uzagalnennja ta zastosuvannja. Lviv.: LNU im. Ivana Franka, 2014. -152 s.

Received 05.12.2019. Accepted 11.12.2019.

\section{Математичне моделювання узагальненої задачі теплообміну тіл півсферичної форми}

Побудована математична модель розрахунку полів температурних полів в півсферичному тілі, з урахуванням кінцевої швидкості поширення тепла, яке обертається, у вигляді крайової задачі математичної фізики для гіперболічного рівняння теплопровідності. Побудоване інтегральне перетворення для двовимірного кінцевого простору, із застосуванням якого знайдено температурне поле у вигляді збіжних рядів по функціям Фур' $\epsilon$.

Mathematic simulation of generalized problem of heat-exchange in the bodies with hemispherical shape

A mathematical model for calculating the fields of temperature fields in a hemispherical body is constructed, taking into account the final velocity of rotating heat propagation, in the form of a boundary value problem of mathematical physics for the hyperbolic equation of thermal conductivity. An integral transformation for a two-dimensional finite space is constructed using a temperature field in the form of convergent Fourier functions.

Бердник Михайло Геннадійович - кандидат фізико-математичних наук, доцент, Національний технічний університет "Дніпровська політехніка", доцент кафедри програмного забезпечення комп’ютерних систем, м.Дніпро, Україна.

Бердник Михаил Геннадьевич - кандидат физико-математических наук, доцент, Национальный технический университет "Днепровская политехника", доцент кафедры программного обеспечения компьютерных систем, м.Днепр, Украина.

Berdnyk Mykhailo Hennadiiovych - Candidate of Physics and Mathematics, assistant professor, National Technical University Dnipro Polytechnic, Senior Lecturer of computer software systems, Dnipro, Ukraine. 